\begin{tabular}{|c|c|}
\hline Title & Kolmogorov-Smirnov type test for generated variables \\
\hline \multicolumn{2}{|l|}{ Sub Title } \\
\hline Author & $\begin{array}{l}\text { 大津，泰介(Ōtsu，Taisuke) } \\
\text { 谷口，豪(Go, Taniguchi) }\end{array}$ \\
\hline Publisher & Keio Economic Observatory Sangyo Kenkyujo \\
\hline Publication year & 2019 \\
\hline Jtitle & KEO discussion paper No.148 (2019. 7) \\
\hline \multicolumn{2}{|l|}{ JaLC DOI } \\
\hline Abstract & $\begin{array}{l}\text { Distribution homogeneity testing, particularly based on the Kolmogorov-Smirnov statistic, has } \\
\text { been applied in various empirical studies. In empirical economic analysis, it is often the case that } \\
\text { economic variables of interest are obtained as fitted values or residuals of preliminary model fits, } \\
\text { called generated variables. In this paper, we extend the Kolmogorov-Smirnov type homogeneity } \\
\text { test to accommodate such generated variables, and propose an asymptotically valid bootstrap } \\
\text { procedure. A small simulation study illustrates that it is crucial for reliable inference to account } \\
\text { for estimation errors in the generated variables. The proposed method is applied to compare the } \\
\text { total factor productivities across different countries. }\end{array}$ \\
\hline \multicolumn{2}{|l|}{ Notes } \\
\hline Genre & Technical Report \\
\hline URL & $\begin{array}{l}\text { https://koara.lib.keio.ac.jp/xoonips/modules/xoonips/detail.php?koara_id=AA10715861-0000014 } \\
\text { 8-0001 }\end{array}$ \\
\hline
\end{tabular}

慶應義塾大学学術情報リポジトリ(KOARA)に掲載されているコンテンツの著作権は、それぞれの著作者、学会または出版社/発行者に帰属し、その権利は著作権法によって 保護されています。引用にあたっては、著作権法を遵守してご利用ください。

The copyrights of content available on the KeiO Associated Repository of Academic resources (KOARA) belong to the respective authors, academic societies, or publishers/issuers, and these rights are protected by the Japanese Copyright Act. When quoting the content, please follow the Japanese copyright act. 
Kolmogorov-Smirnov type test for generated variables

Taisuke Otsu and Go Taniguchi

July 21, 2019

KEO Discussion Paper No. 148 
KEO Discussion Paper No. 148

\title{
KOLMOGOROV-SMIRNOV TYPE TEST FOR GENERATED VARIABLES
}

\author{
TAISUKE OTSU AND GO TANIGUCHI
}

\begin{abstract}
Distribution homogeneity testing, particularly based on the Kolmogorov-Smirnov statistic, has been applied in various empirical studies. In empirical economic analysis, it is often the case that economic variables of interest are obtained as fitted values or residuals of preliminary model fits, called generated variables. In this paper, we extend the KolmogorovSmirnov type homogeneity test to accommodate such generated variables, and propose an asymptotically valid bootstrap procedure. A small simulation study illustrates that it is crucial for reliable inference to account for estimation errors in the generated variables. The proposed method is applied to compare the total factor productivities across different countries.
\end{abstract}

\section{INTRODUCTION}

In various areas of empirical studies, researchers are often interested in testing homogeneity of distributions across different samples. The most popular approach for distribution homogeneity testing is based on the Kolmogorov-Smirnov statistic, which is obtained as the largest discrepancy of the empirical distribution functions by these samples, and statistical theory of the Kolmogorov-Smirnov test is well known (e.g., Lehmann and Romano, 2005). An obvious premise behind this statistical theory is that the samples of interest are all observable.

In empirical economic analysis, however, it is often the case that researchers are interested in the distributions of latent or theoretical variables, which are unobservable but may be estimated by observable data. Such variables, called generated variables, are often obtained as fitted values or residuals of preliminary regression fitting. Common examples include expected values of prices or sales, total factor productivity, relative quality of firms, among others. A major econometric issue of use of generated variables is that its statistical inference requires to account for estimation errors contained in the generated variables. Pagan (1984) investigated how to modify standard errors for regression analysis using generated regressors. Hahn and Ridder (2013) studied standard error formulae for semiparametric estimators that involve generated variables. Matsushita and Otsu (2016) proposed a likelihood-based inference method for semiparametric models with generated variables.

In this paper, we consider distribution homogeneity testing of generated variables. The test statistic is the Kolmogorov-Smirnov statistic using the generated variables. However, the null distribution is different from the conventional one due to the estimation errors to construct the generated variables, and takes a somewhat complicated form. Therefore, we propose a bootstrap procedure to compute the critical value for the Kolmogorov-Smirnov statistic. A 
key ingredient of our bootstrap procedure is to recenter for the bootstrap statistic to impose the null hypothesis (cf. Whang, 2001). By adapting the asymptotic theory developed in Linton, Maasoumi and Whang (2005), the asymptotic validity of our bootstrap procedure can be established.

The proposed homogeneity test is illustrated by Monte Carlo simulation and an empirical application. Our simulation study illustrates that it is crucial for reliable inference to account for estimation errors in the generated variables. Especially the conventional Kolmogorov-Smirnov test may exhibit severe size distortions. Also our empirical application on comparisons of the total factor productivities across different countries illustrate usefulness of the proposed test.

\section{Distribution homogeneity test}

Consider scalar latent variables $X_{k i}$ for $k=1, \ldots, K$, which are not directly observable. Suppose that the latent variables are specified as $X_{k i}=g\left(Z_{k i}, \theta_{k 0}\right)$, where $g$ is a known function up to the unknown parameters $\theta_{k 0}$ and $Z_{k i}$ is a vector of observables. Let $F_{k}$ be the cumulative distribution function of $X_{k}$ for $k=1, \ldots, K$. We wish to conduct hypothesis testing on distribution homogeneity of generated variables

$$
H_{0}: F_{1}(x)=\cdots=F_{K}(x) \text { for all } x
$$

against $H_{1}: H_{0}$ is false.

If $\left\{X_{k i}\right\}_{i=1}^{n}$ is directly observable, then various homogeneity tests are available in the literature. In this paper we focus on the situation where some estimates $\hat{\theta}_{k}$ for the parameters $\theta_{k 0}$ are available to the researcher so that the generated variables $\hat{X}_{k i}=g\left(Z_{k i}, \hat{\theta}_{k}\right)$ can be constructed for $i=1, \ldots, n$. Typical examples of generated variables are fitted values $\left(\hat{X}_{k i}=Z_{k i}^{\prime} \hat{\theta}_{k 0}\right)$ and residuals $\left(\hat{X}_{k i}=Z_{k i}^{(1)}-Z_{k i}^{(2) \prime} \hat{\theta}_{k 0}\right)$ by preliminary OLS linear regression fitting. Let $\hat{F}_{k}(x)=n^{-1} \sum_{i=1}^{n} \mathbb{I}\left\{\hat{X}_{k i} \leq x\right\}$ be the empirical distribution function based on the generated variables $\left\{\hat{X}_{k i}\right\}_{i=1}^{n}$, where $\mathbb{I}\{\cdot\}$ is the indicator function. $\hat{F}_{k}(x)$ is a consistent estimator of $F_{k}(x)$ under mild regularity conditions as far as $\hat{\theta}_{k}$ is consistent for $\theta_{k 0}$. In order to test the distribution homogeneity hypothesis $H_{0}$, we employ the Kolmogorov-Smirnov type statistics

$$
\begin{aligned}
K S_{1} & =\max _{k \neq l} \sup _{x \in \mathcal{X}} \sqrt{n}\left|\hat{F}_{k}(x)-\hat{F}_{l}(x)\right| \\
K S_{2} & =\max _{k} \sup _{x \in \mathcal{X}} \sqrt{n}\left|\hat{F}_{k}(x)-\frac{1}{K} \sum_{l=1}^{K} \hat{F}_{l}(x)\right|,
\end{aligned}
$$

where $\mathcal{X}$ is a given set. The first statistic $K S_{1}$ is the maximal pairwise sup-norm distance of the empirical distributions $\left\{\hat{F}_{k}(\cdot)\right\}_{k=1}^{K}$. The second statistic $K S_{2}$ is obtained by the maximal deviation from the average $K^{-1} \sum_{l=1}^{K} \hat{F}_{l}(x)$, which is equivalent to the empirical distribution by the pooled sample $\left\{\hat{X}_{k i}\right\}_{i=1}^{n}$ over $k=1, \ldots, K$. When the number of different samples $K$ is large, $K S_{2}$ is computationally more attractive. 
The limiting distributions of the Kolmogorov-Smirnov statistics under the null hypothesis $H_{0}$ are obtained as follows.

Proposition. For each $k=1, \ldots, K$, suppose

(i): $\left\{Z_{k i}\right\}_{i=1}^{n}$ is a strictly stationary and $\alpha$-mixing sequence with the mixing coefficient $\alpha(m)=O\left(m^{-a}\right)$ for some $a>\max \{(q-1)(q+1), 1+2 / r\}$, where $q$ is an even integer satisfying $q>3\left(\max \left\{\operatorname{dim} \theta_{1}, \ldots, \operatorname{dim} \theta_{K}\right\}+1\right) / 2$ and $r$ appears in (ii) below. For a neighborhood $\mathcal{N}_{k}$ around $\theta_{k 0}, F_{k}\left(x, \theta_{k}\right)=P\left\{g\left(Z_{k i}, \theta_{k}\right) \leq x\right\}$ is differentiable on $\theta_{k} \in$ $\mathcal{N}_{k}, \sup _{x \in \mathcal{X}, \theta:\left|\theta-\theta_{k 0}\right| \leq \delta_{n}}\left|\partial F_{k}\left(x, \theta_{k}\right) / \partial \theta_{k}-\partial F_{k}\left(x, \theta_{k 0}\right) / \partial \theta_{k}\right| \rightarrow 0$ for any positive sequence $\delta_{n} \rightarrow 0, \sup _{x \in \mathcal{X}}\left|\partial F_{k}\left(x, \theta_{k 0}\right) / \partial \theta_{k}\right|<\infty$, and $E\left[\sup _{\theta_{k} \in \mathcal{N}_{k}}\left|\partial g\left(Z_{k i}, \theta_{k}\right) / \partial \theta_{k}\right|^{2}\right]<\infty$;

(ii): the estimator $\hat{\theta}_{k}$ satisfies $\sqrt{n}\left(\hat{\theta}_{k}-\theta_{k 0}\right)=n^{-1 / 2} \sum_{i=1}^{n} \psi_{k}\left(Z_{k i}, \theta_{k 0}\right)+o_{p}(1)$ with $E\left[\psi_{k}\left(Z_{k i}, \theta_{k 0}\right)\right]=$ 0 and $E\left[\left|\psi_{k}\left(Z_{k i}, \theta_{k 0}\right)\right|^{2+r}\right]<\infty$ for some $r>0$.

Then under $H_{0}$,

$$
\begin{aligned}
& K S_{1} \stackrel{d}{\rightarrow} \max _{k \neq l} \sup _{x \in \mathcal{X}}\left|\nu_{k l}(x)+\frac{\partial F_{k}\left(x, \theta_{k 0}\right)}{\partial \theta_{k}^{\prime}} \xi_{k}-\frac{\partial F_{l}\left(x, \theta_{l 0}\right)}{\partial \theta_{l}^{\prime}} \xi_{l}\right|, \\
& K S_{2} \stackrel{d}{\rightarrow} \max _{k} \sup _{x \in \mathcal{X}}\left|\frac{1}{K} \sum_{l=1}^{K}\left\{\nu_{k l}(x)+\frac{\partial F_{k}\left(x, \theta_{k 0}\right)}{\partial \theta_{k}^{\prime}} \xi_{k}-\frac{\partial F_{l}\left(x, \theta_{l 0}\right)}{\partial \theta_{l}^{\prime}} \xi_{l}\right\}\right|,
\end{aligned}
$$

where $\left(\nu_{k l}(\cdot), \xi_{k}^{\prime}, \xi_{l}^{\prime}\right)$ is a mean zero Gaussian process with the covariance kernel $C_{k l}\left(x_{1}, x_{2}\right)=$ $\lim _{n \rightarrow \infty} n E\left[S_{k l, n}\left(x_{1}\right) S_{k l, n}\left(x_{2}\right)^{\prime}\right]$

$$
\begin{aligned}
S_{k l, n}(x) & =\left(\hat{\nu}_{k}\left(x, \theta_{k 0}\right)-\hat{\nu}_{l}\left(x, \theta_{l 0}\right), \frac{1}{\sqrt{n}} \sum_{i=1}^{n} \psi_{k}\left(Z_{k i}, \theta_{k 0}\right)^{\prime}, \frac{1}{\sqrt{n}} \sum_{i=1}^{n} \psi_{l}\left(Z_{l i}, \theta_{l 0}\right)^{\prime}\right)^{\prime}, \\
\hat{\nu}_{k}\left(x, \theta_{k}\right) & =n^{-1 / 2} \sum_{i=1}^{n}\left\{\mathbb{I}\left\{g\left(Z_{k i}, \theta_{k}\right) \leq x\right\}-F_{k}\left(x, \theta_{k}\right)\right\} .
\end{aligned}
$$

If $X_{k i}$ 's are directly observable, then the limiting null distributions of $K S_{1}$ and $K S_{2}$ reduce to $\max _{k \neq l} \sup _{x \in \mathcal{X}}\left|\nu_{k l}(x)\right|$ and $\max _{k} \sup _{x \in \mathcal{X}}\left|K^{-1} \sum_{l=1}^{K} \nu_{k l}(x)\right|$, respectively. Thus, the terms in (2) containing $\xi_{k}$ 's are considered as correction terms to account for the estimation errors of the parameter estimators, $\hat{\theta}_{k}$ 's.

The assumptions for this proposition are adapted from Linton, Maasoumi and Whang (2005) to our setup. Also, the proof is obtained by modifying that of Linton, Maasoumi and Whang (2005, Theorem 1). Here we sketch the proof for the case of $K S_{1}$. The proof for $K S_{2}$ is similar. Under $H_{0}$ (i.e., $\left.F_{k}\left(x, \theta_{k 0}\right)-F_{l}\left(x, \theta_{l 0}\right)=0\right), K S_{1}$ can be written as

$$
K S_{1}=\max _{k \neq l} \sup _{x \in \mathcal{X}}\left|\hat{\nu}_{k}\left(x, \hat{\theta}_{k}\right)-\hat{\nu}_{l}\left(x, \hat{\theta}_{l}\right)+\sqrt{n}\left\{F_{k}\left(x, \hat{\theta}_{k}\right)-F_{l}\left(x, \hat{\theta}_{l}\right)\right\}-\sqrt{n}\left\{F_{k}\left(x, \theta_{k 0}\right)-F_{l}\left(x, \theta_{l 0}\right)\right\}\right| .
$$


By Linton, Maasoumi and Whang (2005, Lemmas 2 and 3),

$$
\begin{aligned}
& \sup _{x \in \mathcal{X}}\left|\hat{\nu}_{k}\left(x, \hat{\theta}_{k}\right)-\hat{\nu}_{k}\left(x, \theta_{k 0}\right)\right| \stackrel{p}{\rightarrow} 0, \\
& \sup _{x \in \mathcal{X}} \sqrt{n}\left|F_{k}\left(x, \hat{\theta}_{k}\right)-F_{k}\left(x, \theta_{k 0}\right)-\frac{\partial F_{k}\left(x, \theta_{k 0}\right)}{\partial \theta_{k}^{\prime}} \frac{1}{n} \sum_{i=1}^{n} \psi_{k}\left(Z_{k i}, \theta_{k 0}\right)\right| \stackrel{p}{\rightarrow} 0,
\end{aligned}
$$

for $k=1, \ldots, K$. Therefore, we obtain

$$
K S_{1}=\max _{k \neq l} \sup _{x \in \mathcal{X}}\left|\begin{array}{l}
\hat{\nu}_{k}\left(x, \theta_{k 0}\right)-\hat{\nu}_{l}\left(x, \theta_{l 0}\right) \\
+\frac{\partial F_{k}\left(x, \theta_{k 0}\right)}{\partial \theta_{k}^{\prime}} \frac{1}{n} \sum_{i=1}^{n} \psi_{k}\left(Z_{k i}, \theta_{k 0}\right)-\frac{\partial F_{l}\left(x, \theta_{l 0}\right)}{\partial \theta_{l}^{\prime}} \frac{1}{n} \sum_{i=1}^{n} \psi_{l}\left(Z_{l i}, \theta_{l 0}\right)
\end{array}\right|+o_{p}(1),
$$

and the conclusion in (2) follows by the weak convergence of the empirical process $\hat{\nu}_{k}\left(x, \theta_{k 0}\right)$ and continuous mapping theorem.

We can also show that the Kolmogorov-Smirnov tests based on $K S_{1}$ and $K S_{2}$ are consistent under fixed alternative hypotheses and have non-trivial power under the local alternative hypotheses at the rate of $n^{-1 / 2}$.

Due to the correction terms associated with $\xi_{k}$ 's, the limiting null distributions of the Kolmogorov-Smirnov type statistics are not asymptotically pivotal and somewhat complicated. Thus, we suggest the following bootstrap procedure to approximate the critical values.

\section{Bootstrap procedure for critical values:}

(1) Draw the bootstrap resample $\left\{Z_{1 i}^{*}, \ldots, Z_{K i}^{*}\right\}_{i=1}^{n}$ from the joint empirical distribution of $\left\{Z_{1 i}, \ldots, Z_{K i}\right\}_{i=1}^{n}$, and compute the estimator $\left\{\hat{\theta}_{1}^{*}, \ldots, \hat{\theta}_{K}^{*}\right\}$ by using $\left\{Z_{1 i}^{*}, \ldots, Z_{K i}^{*}\right\}_{i=1}^{n}$.

(2) Then compute the recentered bootstrap statistics

$$
\begin{gathered}
K S_{1}^{*}=\max _{k \neq l} \sup _{x} \sqrt{n}\left|\hat{F}_{k}^{*}(x)-\hat{F}_{l}^{*}(x)-\left\{\hat{F}_{k}(x)-\hat{F}_{l}(x)\right\}\right|, \\
K S_{2}^{*}=\max _{k} \sup _{x} \sqrt{n}\left|\hat{F}_{k}^{*}(x)-\frac{1}{K} \sum_{l=1}^{K} \hat{F}_{l}^{*}(x)-\left\{\hat{F}_{k}(x)-\frac{1}{K} \sum_{l=1}^{K} \hat{F}_{l}(x)\right\}\right|,
\end{gathered}
$$

where $\hat{F}_{k}^{*}(x)=n^{-1} \sum_{i=1}^{n} \mathbb{I}\left\{g\left(Z_{k i}^{*}, \hat{\theta}_{k}^{*}\right) \leq x\right\}$.

(3) Repeat (2) $B$ times to obtain $\left\{K S_{1, b}^{*}\right\}_{b=1}^{B}$ or $\left\{K S_{2, b}^{*}\right\}_{b=1}^{B}$. Their quantiles provide the bootstrap critical values of $K S_{1}$ or $K S_{2}$ to test $H_{0}$.

Note that the recentering in $K S_{1}^{*}$ or $K S_{2}^{*}$ is crucial to impose the null hypothesis $H_{0}$. The idea of recentering was suggested in the literature by Hall and Horowitz (1996), Whang (2001), and Linton, Maasoumi and Whang (2005), for example. To obtain an intuition, consider the bootstrap counterpart $K S_{1}^{*}$. We can show that $K S_{1}^{*}$ satisfies

$$
K S_{1}^{*}=\max _{k \neq l} \sup _{x \in \mathcal{X}}\left|\begin{array}{l}
\hat{\nu}_{k}^{*}\left(x, \hat{\theta}_{k}\right)-\hat{\nu}_{l}^{*}\left(x, \hat{\theta}_{l}\right) \\
+\frac{\partial F_{k}\left(x, \theta_{k 0}\right)}{\partial \theta_{k}^{\prime}} \frac{1}{n} \sum_{i=1}^{n} \psi_{k}\left(Z_{k i}^{*}, \hat{\theta}_{k}\right)-\frac{\partial F_{l}\left(x, \theta_{l 0}\right)}{\partial \theta_{l}^{\prime}} \frac{1}{n} \sum_{i=1}^{n} \psi_{l}\left(Z_{l i}^{*}, \hat{\theta}_{l}\right)
\end{array}\right|+o_{p}(1),
$$

conditional on $\left\{Z_{1 i}, \ldots, Z_{K i}\right\}_{i=1}^{n}$ with probability one, where $\hat{\nu}_{k}^{*}\left(x, \theta_{k}\right)=n^{-1 / 2} \sum_{i=1}^{n}\left[\mathbb{I}\left\{g\left(Z_{k i}^{*}, \theta_{k}\right) \leq x\right\}-\mathbb{I}\left\{g\left(Z_{k i}, \theta_{k}\right) \leq x\right\}\right]$. This expression is analogous to 
(3) and guarantees the asymptotic validity of our bootstrap procedure. Without recentering in $K S_{1}^{*}$, we would have additional terms in the above expansion of $K S_{1}^{*}$ that may diverge.

\section{Simulation}

To illustrate the finite sample performance of our Kolmogorov-Smirnov type tests, we conduct a small simulation study. We consider two regression models

$$
Y_{1 i}=1+(1,1) X_{1 i}+e_{1 i}, \quad Y_{2 i}=1+(1,1) X_{2 i}+e_{2 i},
$$

for $i=1, \ldots, n$, where $X_{1 i}$ and $X_{2 i}$ are bivariate regressors generated from

$$
X_{1 i} \sim N\left(\left(\begin{array}{l}
0 \\
0
\end{array}\right),\left(\begin{array}{cc}
1 & 0.5 \\
0.5 & 1
\end{array}\right)\right), \quad X_{2 i} \sim N\left(\left(\begin{array}{l}
0 \\
0
\end{array}\right),\left(\begin{array}{cc}
1 & 0.7 \\
0.7 & 1
\end{array}\right)\right) .
$$

In this setup, we consider homogeneity testing of the distribution functions of the error terms $e_{1}$ and $e_{2}$, i.e.,

$$
H_{0}: F_{e_{1}}(t)=F_{e_{2}}(t) \text { for all } t,
$$

based on the OLS residuals $\hat{e}_{1 i}$ and $\hat{e}_{2 i}$ for the regressions from $Y_{1}$ on $X_{1}$ and $Y_{2}$ on $X_{2}$, respectively.

For the test statistic $K S_{1}$ in (1), we compare the proposed bootstrap procedure with the conventional Kolmogorov-Smirnov critical value that does not take into account for the estimation errors $\hat{e}_{1 i}-e_{1 i}$ and $\hat{e}_{2 i}-e_{2 i}$. Note that the conventional critical value is asymptotically invalid and is used to illustrate importance of accommodating the estimation errors for the generated variables.

To evaluate the size properties, we consider three distributions of $e_{1}$ and $e_{2}: N(0,1)$, standardized $t(3)$, standardized $\chi^{2}(3)$, and standardized $L N(0,1)$ (log-normal generated by $\exp (Z)$ for $Z \sim N(0,1))$. For the sample size, we consider $n=100,200$, and 500. The number of bootstrap replications is 99 and the number of Monte Carlo replications is 1000. The nominal size is 0.05 . Table 1 presents the rejection frequencies under the null hypotheses. Our bootstrap procedure works well for all cases although it shows under-coverage for most cases. On the other hand, the conventional Kolmogorov-Smirnov critical value clearly fails to control the size for the case of $L N(0,1)$. This indicates that it is crucial to take into account for the estimation errors of the generated variables to conduct homogeneity testing.

We next evaluate power properties of the proposed test. Now the error terms in (4) are generate by

$$
\begin{aligned}
& e_{1 i}=\sqrt{\rho} \epsilon_{0 i}+\sqrt{1-\rho} \epsilon_{1 i}, \\
& e_{2 i}=\sqrt{\rho} \epsilon_{0 i}+\sqrt{1-\rho} \epsilon_{2 i},
\end{aligned}
$$

for $i=1, \ldots, n$, where $\rho \in\{0.0,0.2,0.4\}, \epsilon_{0 i} \sim N(0,1), \epsilon_{1 i} \sim N(0,1)$, and $\epsilon_{2 i}$ follows the standardized $t(3)$, standardized $\chi^{2}(3)$, and standardized $L N(0,1)$. The distributions of $e_{1 i}$ and $e_{2 i}$ become similar as $\rho$ increases. 
TABLE 1. Rejection Frequencies (Size)

\begin{tabular}{c|c|ccc} 
& & \multicolumn{3}{|c}{$n$} \\
$F_{e_{1}}=F_{e_{2}}$ & Method & 100 & 200 & 500 \\
\hline \hline$N(0,1)$ & Bootstrap & .001 & .003 & .006 \\
& Conventional KS & .001 & .001 & .002 \\
\hline$t(3)$ & Bootstrap & .009 & .007 & .013 \\
& Conventional KS & .052 & .064 & .078 \\
\hline$\chi^{2}(3)$ & Bootstrap & .009 & .017 & .018 \\
& Conventional KS & .005 & .072 & .069 \\
\hline$L N(0,1)$ & Bootstrap & .043 & .048 & .053 \\
& Conventional KS & .390 & .441 & .506 \\
\hline
\end{tabular}

Table 2 presents the rejection frequencies under the alternative hypotheses based on 1000 Monte Carlo replications. The proposed bootstrap test shows reasonable power properties when the sample size is large enough. The power of the conventional Kolmogorov-Smirnov for $\operatorname{LN}(0,1)$ is spurious because of severe over-rejection under the null hypothesis. Also, as $\rho$ increases, the power of the conventional Kolmogorov-Smirnov deteriorates faster than the bootstrap test. Finally, although the results are not reported, we find that the bootstrap statistic without recentering has zero power for all cases.

TABLE 2. Rejection Frequencies (Power)

\begin{tabular}{c|c|ccc|ccc|ccc} 
& & \multicolumn{3}{|c|}{$n=100$} & \multicolumn{3}{c|}{$n=200$} & \multicolumn{3}{c}{$n=500$} \\
\hline \multirow{3}{*}{$F_{\epsilon_{2}}$} & Method & \multicolumn{4}{|c|}{$\rho$} & $\rho$ & \multicolumn{4}{c|}{$\rho$} \\
\hline \hline$t(3)$ & Bootstrap & .087 & .024 & .007 & .378 & .108 & .026 & .946 & .579 & .191 \\
& Conventional KS & .128 & .027 & .007 & .495 & .107 & .022 & .987 & .539 & .084 \\
\hline$\chi^{2}(3)$ & Bootstrap & .114 & .045 & .016 & .605 & .243 & .061 & 1 & .883 & .416 \\
& Conventional KS & .255 & .063 & .012 & .779 & .239 & .049 & 1 & .841 & .255 \\
\hline$L N(0,1)$ & Bootstrap & .312 & .195 & .038 & .780 & .719 & .237 & .997 & .997 & .901 \\
& Conventional KS & .979 & .384 & .067 & 1 & .884 & .260 & 1 & 1 & .855 \\
\hline
\end{tabular}

\section{EMPiRiCAl illustration: TOTAL FACTOR PRODUCTIVITy}

We apply our Kolmogorov-Smirnov type test to compare the total factor productivities among different countries. Following Solow's (1957) classical approach, we specify the production function as $Y_{t}=A_{t} K_{t}^{\alpha} L_{t}^{\beta}$, where $Y_{t}$ is total output, $K_{t}$ and $L_{t}$ are capital and labor inputs, respectively, and $A_{t}$ is total factor productivity. Solow (1957) specified the production function as $Y_{t}=A_{t} F\left(K_{t}, L_{t}\right)$, where $Y_{t}$ is total output, $K_{t}$ and $L_{t}$ are capital and labor inputs, respectively, and $A_{t}$ is total factor productivity. ${ }^{1}$ If we specify $F$ by the Cobb-Douglas function $F\left(K_{t}, L_{t}\right)=K_{t}^{\alpha} L_{t}^{\beta}$, we can derive

$$
\Delta \log \left(A_{t}\right)=\Delta \log \left(Y_{t}\right)-\alpha \Delta \log \left(K_{t}\right)-\beta \Delta \log \left(L_{t}\right) .
$$

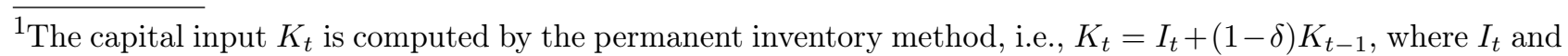
$\delta$ are gross investment and depreciation rate, respectively. We use the data on gross fixed capital formulation for $I_{t}$, and set the depreciation rate as $\delta=0.05$. The initial capital stock $K_{0}$ is calculated by $K_{0}=I_{0} / \delta$.
} 
We use the datasets offered by the Federal Reserve Bank of St. Louis (FRED), OECD, United Nations Statistics Division, and World Bank (see Table 3).

TABLE 3. List of variables

\begin{tabular}{c|c}
\hline Variable & Measurement \\
\hline \hline$Y$ & Gross domestic products in the country (World Bank) \\
\hline$I$ & Gross fixed capital formation in the country (FRED) \\
\hline$L$ & $\begin{array}{c}\text { Population (OECD) } \\
\end{array}$ \\
& $\times$ Percentage of working age population (OECD) \\
\hline
\end{tabular}

Using these data, we calculate the annual growth rate of total factor productivity from 1971 to 2014 for 15 countries (Australia, Austria, Belgium, Canada, Denmark, France, Germany, Italy, Japan, Netherlands, Norway, South Korea, Sweden, United Kingdom, and United States). In particular, $\Delta \log \left(A_{t}\right)$ is estimated by evaluating $\alpha$ and $\beta$ in (5) with the OLS estimator.

We apply the Kolmogorov-Smirnov type test for generated variables to test homogeneity of pairs of distributions of $\Delta \log \left(A_{t}\right)$ from 15 countries. As in the simulation study, we compare our bootstrap method with the conventional Kolmogorov-Smirnov critical value that does not take into account for the estimation errors. The results are presented in Table 4. We can see that the conclusions of the tests are different for several cases (indicated by bold letters). Also those conclusions can be different in either ways. For reliable inference, it is critical to incorporate estimation errors for generated variables as in our bootstrap method. 


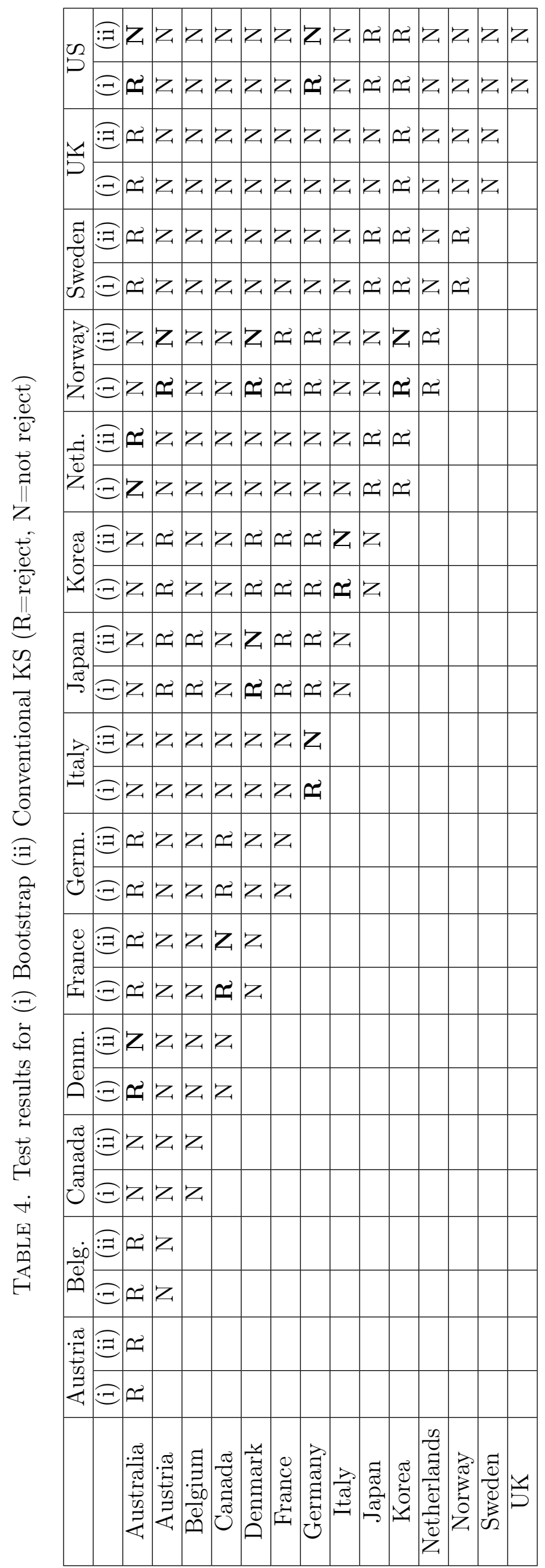




\section{REFERENCES}

[1] Hahn, J. and G. Ridder (2013) Asymptotic variance of semiparametric estimators with generated regressors, Econometrica, 81, 315-340.

[2] Hall, P. and J. L. Horowitz (1996) Bootstrap critical values for tests based on generalized-method-of-moments estimators, Econometrica, 64, 891-916.

[3] Lehmann, E. L. and J. P. Romano (2005) Testing Statistical Hypotheses, 3rd ed., Springer.

[4] Linton, O., Maasoumi, E. and Y.-J. Whang (2005) Consistent testing for stochastic dominance under general sampling schemes, Review of Economic Studies, 72, 735-765.

[5] Matsushita, Y. and T. Otsu (2016) Likelihood inference on semiparametric models with generated regressors, Working paper.

[6] Pagan, A. (1984) Econometric issues in the analysis of regressions with generated regressors, International Economic Review, 25, 221-247.

[7] Solow, R. M. (1957) Technical change and the aggregate production function, Review of Economics and Statistics, 39, 312-320.

[8] Whang, Y.-J. (2001) Consistent specification testing for conditional moment restrictions, Economics Letters, $71,299-306$.

Department of Economics, London School of Economics, Houghton Street, London, WC2A 2AE, UK, and Keio Economic Observatory (KEO), 2-15-45 Mita, Minato-Ku, Tokyo 108-8345, JAPAN.

Email address: t.otsu@lse.ac.uk

Research Center for Policy and Economy, Mitsubishi Research Institute, Inc., 10-3, NagatACHO 2-CHOME, CHIYOdA-KU, TOKYo 100-8141, Japan.

Email address: go_taniguchi@mri.co.jp 